\title{
Can cangrelor bridge the gap to cardiac surgery?
}

Cangrelor, an intravenous reversible inhibitor of the P2Y $\mathrm{Y}_{12}$ platelet receptor, could be used to 'bridge' patients from irreversible antiplatelet therapy to cardiac surgery, suggest Dominick J. Angiolillo (University of Florida, USA) and colleagues in a report published in JAMA.

Dual antiplatelet therapy (aspirin and an irreversible thienopyridine $\mathrm{P}_{2} \mathrm{Y}_{12}$ receptor inhibitor) is commonly used to reduce the risk of cardiovascular events in patients with acute coronary syndromes or those undergoing percutaneous coronary intervention. However, as this treatment increases the risk of bleeding complications, cessation of therapy is recommended 5-7 days prior to surgery to allow recovery of platelet function. Premature discontinuation of antiplatelet therapy increases the risk of ischemic complications. Therefore, presurgery antithrombotic strategies, which both minimize the risk of atherothrombotic events and allow normal hemostasis to be maintained during surgery, are required. Reversible antiplatelet therapy could meet these criteria.

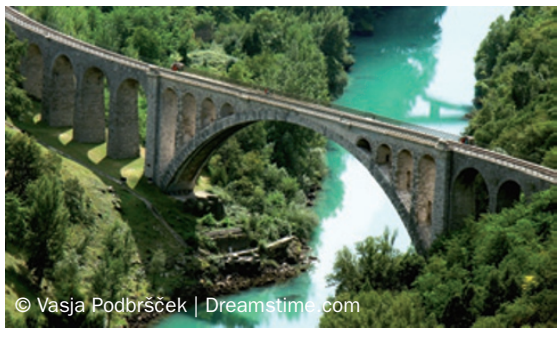

Angiolillo and colleagues evaluated a $0.75 \mu \mathrm{g} / \mathrm{kg} / \mathrm{min}$ infusion of cangrelor for bridging thienopyridine-treated patients with an acute coronary syndrome, or coronary stent, to CABG surgery. Patients were randomly assigned to receive cangrelor $(n=106)$ or placebo $(n=101)$ for at least $48 \mathrm{~h}$ following discontinuation of thienopyridine therapy. Cangrelor infusion was stopped 1-6h prior to surgery, and platelet function assessed before, during, and after infusion using the point-of-care platelet function test.

"A significantly greater proportion of patients treated with cangrelor had low levels of platelet reactivity throughout the entire treatment period compared with placebo," says Angiolillo (98.8\% vs 19.0\%;
RR 5.2, 95\% CI 3.3-8.1, $P<0.001)$. “This occurred without excessive CABG-related bleeding." Presurgery minor bleeding events were more frequent among the cangrelor group. However, platelet function recovered rapidly after infusion cessation and no significant difference was noted in study-defined excessive CABG surgeryrelated bleeding between the placebo and treatment groups.

The trial was not powered to assess differences in bleeding event rates, or if cangrelor treatment prior to $\mathrm{CABG}$ surgery reduced the incidence of ischemic events. However, Angiolillo and colleagues conclude that "intravenous cangrelor is a feasible management strategy in patients waiting for cardiac surgery who require prolonged $\mathrm{P} 2 \mathrm{Y}_{12}$ inhibition after thienopyridine discontinuation."

Ellen F. Carney

Original article Angiolillo, D. J. et al. Bridging antiplatelet therapy with cangrelor in patients undergoing cardiac surgery: a randomized controlled trial. JAMA 307, 265-274 (2012) 